\title{
Mycoplasma pneumoniae in pediatric patients: do macrolide-resistance and/or delayed treatment matter?
}

Tzu-I Yang', Tu-Hsuan Chang., Chun-Yi Lu, Jong-Min Chen', Ping-Ing Lee, Li-Min Huang', Luan-Yin Chang.

\section{Introduction:}

Mycoplasma pneumoniae(MP) is a common pathogen for pneumonia in children especially in the post-pneumococcal conjugate vaccination era, up to $30-40 \%{ }^{1}$ Though self-limted disease was found in the majority of the patients, severe diseases with complication ${ }^{2}$ occured occasionally and was related to immune response. The emergence of macrolide resistance was also reported worldwide, up to $90 \%$ in some area in Asia. It is important to delineate whether macrolide resistance or delayed treatment affects outcome.

\section{Methods:}

We retrospectively collected pediatric patients with clinical suspicion of Mycoplasma pneumoniae infection and confirmed by positive PCR from respiraoty tract specimen subsequently in a tertiary medical center in Taiwan from 1st Jan. 2010 to 30th Jun. 2017. Patients with incomplete or loss of medical record, immunocompromised condition or malignancies were excluded.

\section{Resistant rate and strain}

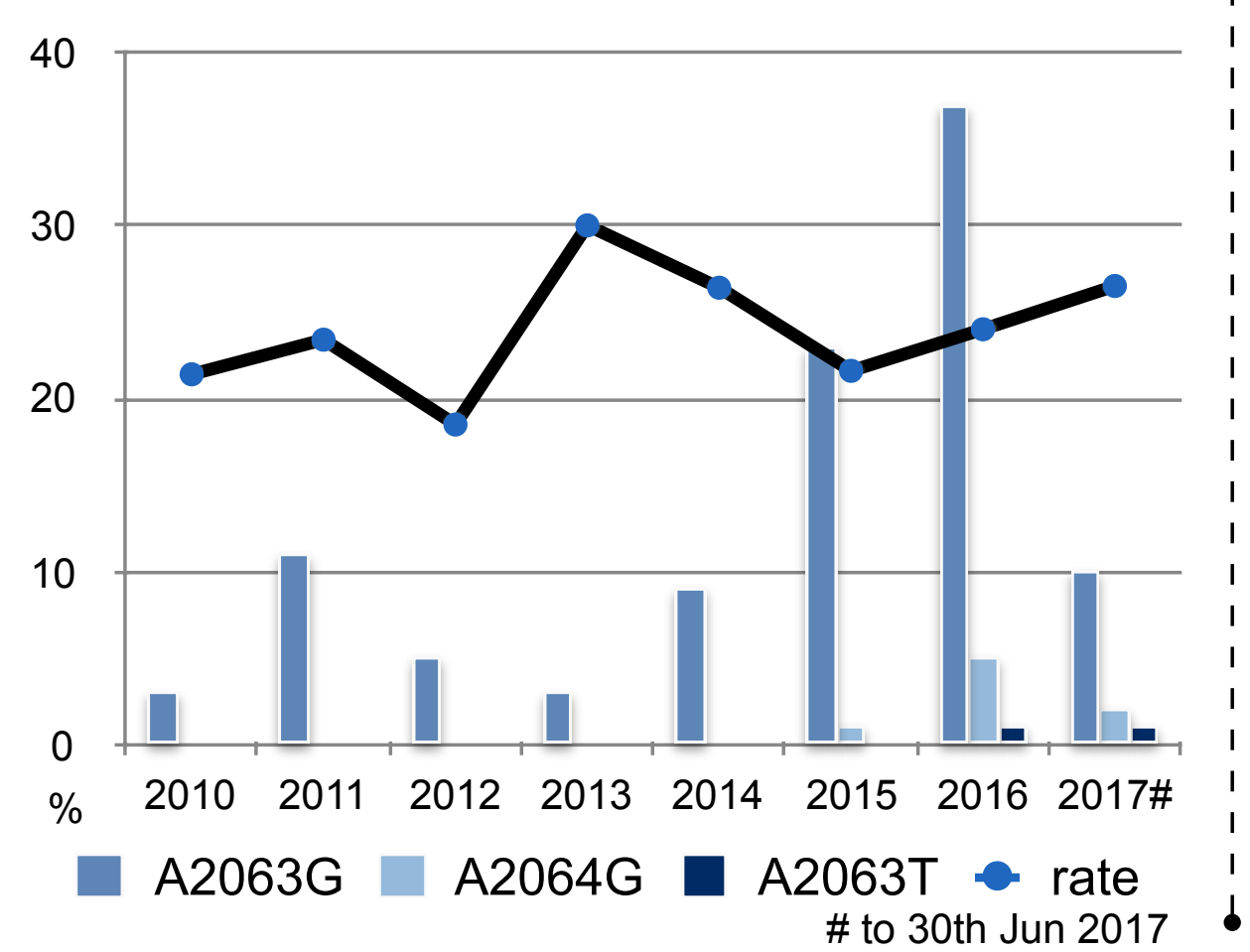

Patients' history, demographics, clinical manifestations, laboratory parameters radiographic findings, MP bacterial load, $\mathrm{Co}$ infection pathogens and treatment for MP were reviewed from medical charts and performed statistical analysis. Polymerase chain reaction assay were performed when clinically suspicion twice weekly, the segment of 4596-4725 nucleotides of cytadhesin P1 gene was targeted for MP detection. Resistant gene was examed by nested PCR which detecting reported sites in 2758-2769 (A2063G/C, A2064G/C, C2617G/A)

\section{Macrolid resistant or not}

30

22.5 15 7.5 7.5 0

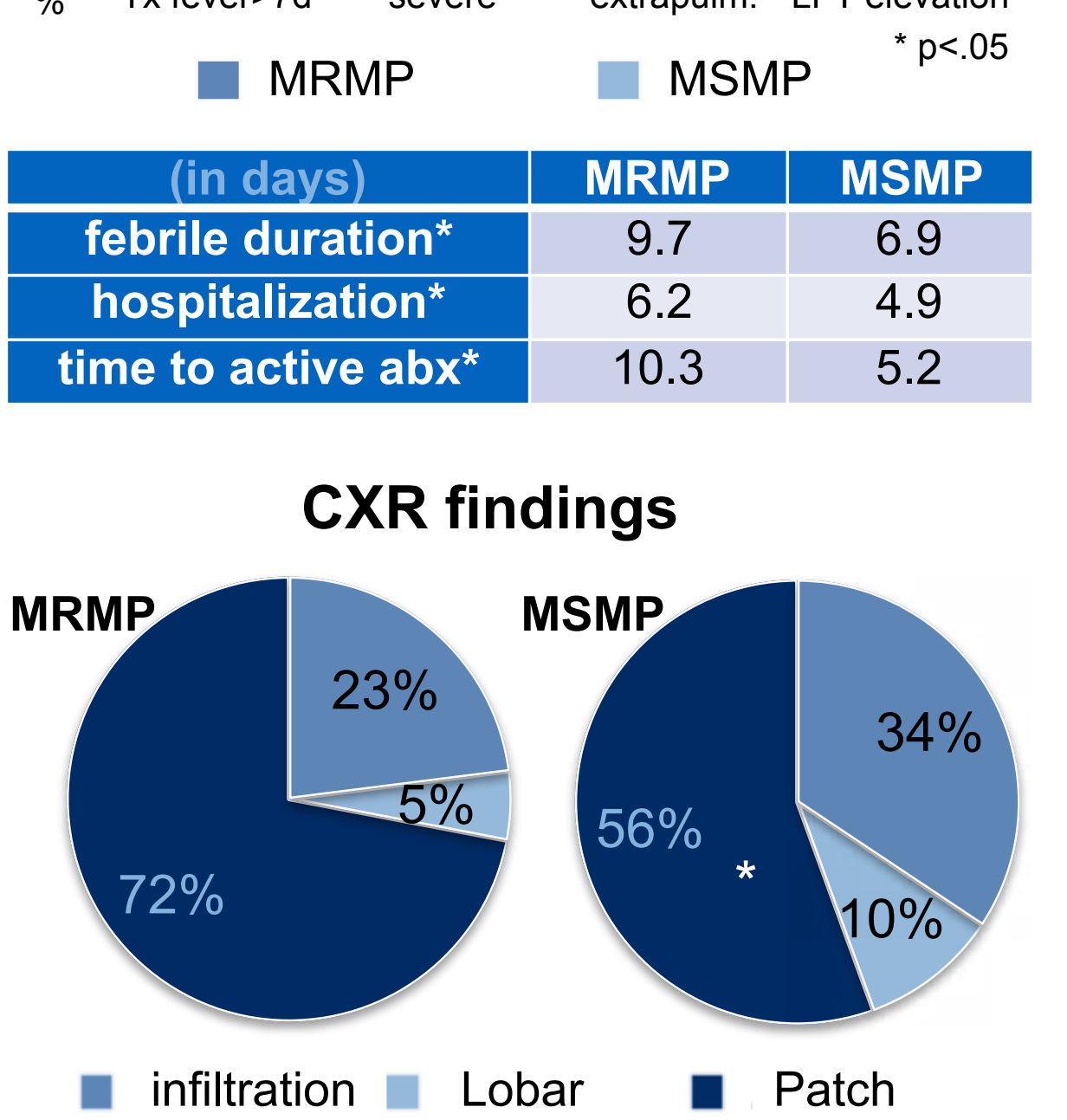

Results:

Among 471 children with positive MP PCR in 2327 tested, the mean age is 6.6 years and $51 \%$ are male. $95 \%$ were diagnosed with pneumonia, $2.5 \%$ had severe disease (respiratory failure needs ventilator support SJS, encephalitis), $15 \%$ had pleural effusion ( $50 \%$ effusion were PCR positive), $17 \%$ had extrapulmonary complications (43 with skin rash, 42 with impaired liver function, and 5 with CNS involvement).

The mean rate of macrolide resistance was $24 \%(19-30 \%)$ and macrolide resistance was related to longer febrile duration, longer hospital stay, lung consolidation and impaired liver function tests. Mean resistant rate among patients age $<2$ were $14 \%$. The medium bacterial load of all cases was $1.5 \times 10^{\circ}$ copies $/ \mathrm{ml}\left(2.6 \times 10_{2}\right.$ to $1 \times 10^{\circ}$ copies $\left./ \mathrm{ml}\right)$. No significant difference between high and low load except patients with load lower than medium had extrapulmonary manifestations and viral coinfection. Overall viral co-infection rate was $7.1 \%$ and bacterial co-infection rate was $4.6 \%$.

\section{Effect of delayed treatment}

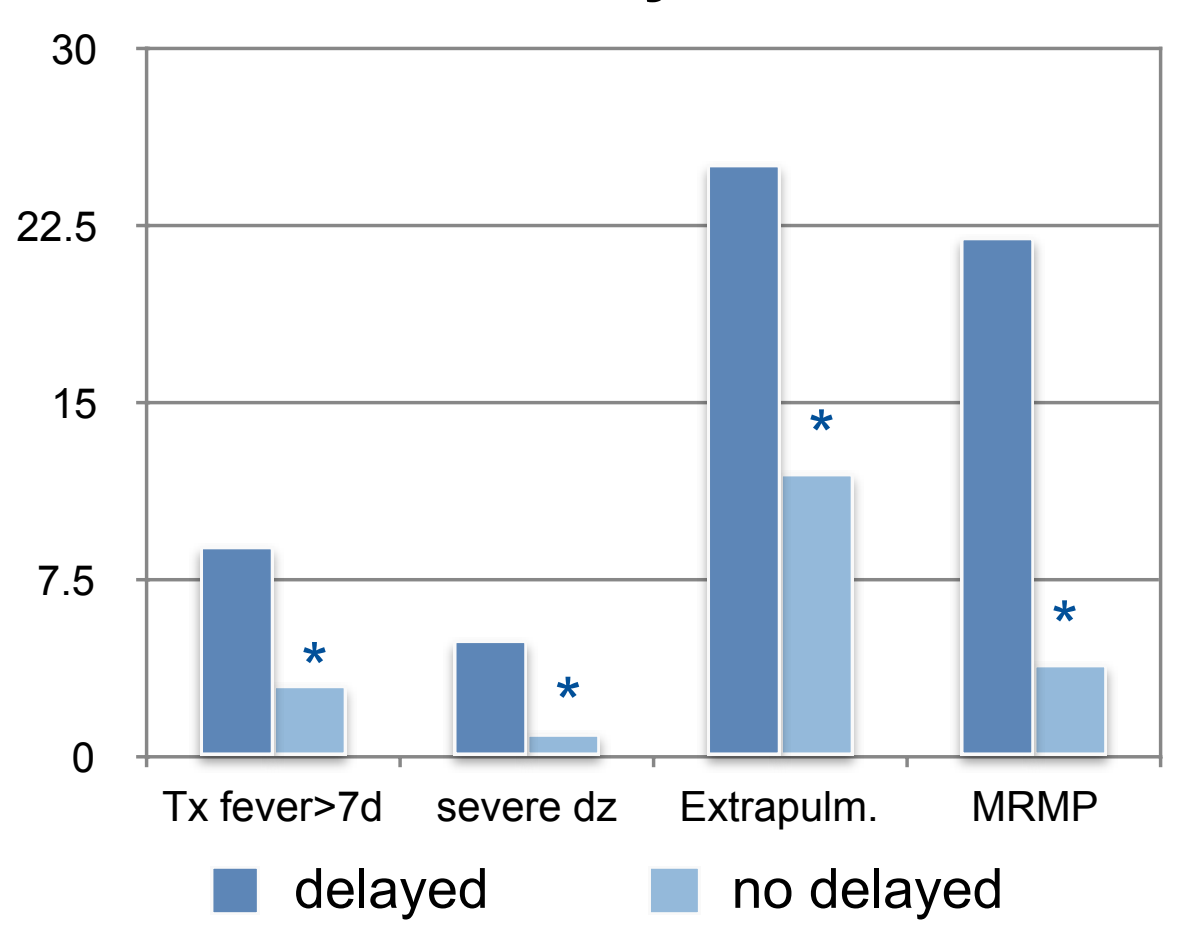

Delayed active treatment (longer than medium, 5.6 days), which related to MRMP was also associated with severe disease, prolonged fever after MP-active treatment, extrapulmonary manifestations and higher CRP. In patients presented with pleural effusion in the clinical course, extrapulmonary manifestation and severe diseases were more often. Second-line antibiotics includes doxycycline and quinolones were prescribed more often, however, pleural effusion it is not associated MRMP.

\section{Conclusions:}

Macrolide resistance was fairly common and might lead to delayed appropriate antibiotic treatment in Taiwan. Delayed appropriate antimicrobial treatment, no matter macrolide resistance or not, was associated with more severe and/or prolonged diseases among pediatric patients with Mycoplasma pneumoniae infection. Early diagnosis of Mycoplasma pneumoniae as well as the awareness of macrolide resistance makes early effective antibiotic treatment possible and may improve clinical outcomes.

\section{With or without pleural effusion}

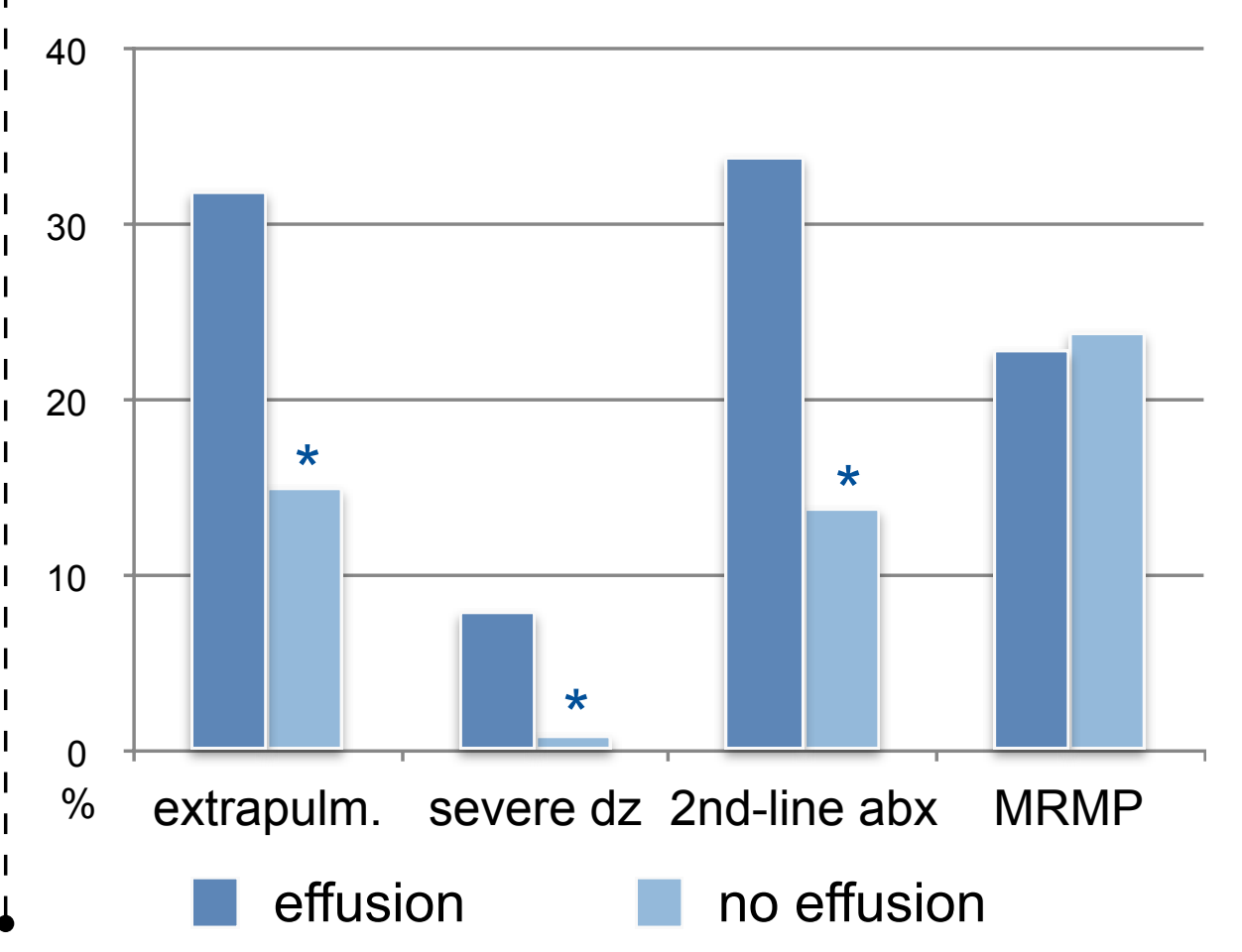

